\title{
Solution of the Inverse Problem of Subsurface Electric Exploration for Horizontally Stratified Medium
}

\author{
A. L. Karchevsky, ${ }^{1}$ Zh. O. Oralbekova, ${ }^{2}$ and K. T. Iskakov ${ }^{3}$ \\ ${ }^{1}$ Sobolev Institute of Mathematics of Siberian Branch of Russian Academy of Sciences, Novosibirsk 630090, Russia \\ ${ }^{2}$ Abai Kazakh National Pedagogical University, Almaty 050010, Kazakhstan \\ ${ }^{3}$ L.N. Gumilyov Eurasian National University, Astana 10008, Kazakhstan
}

Correspondence should be addressed to Zh. O. Oralbekova; oralbekova@bk.ru

Received 25 February 2013; Accepted 27 July 2013

Academic Editor: Abdel-Maksoud A. Soliman

Copyright (C) 2013 A. L. Karchevsky et al. This is an open access article distributed under the Creative Commons Attribution License, which permits unrestricted use, distribution, and reproduction in any medium, provided the original work is properly cited.

In this paper, we offer the generalization of the known technique of the construction of the gradient of the residual functional based on the statement of the conjugate problem for the case when the unknown function is complex valued. The notion of the reference frequency of the medium is introduced. Knowing the value of the reference frequency lets us judge the possibility of simultaneous definition of the dielectric permittivity and conductivity.

\section{Introduction}

The problem of definition of the dielectric permittivity and conductivity is the actual geophysical problem. Theoretical research of inverse problems of geoelectrics and some numerical examples of definition of these functions can be found in [1].

There are many works devoted to the problems of reconstruction of conductivity $\sigma$ and dielectric permittivity $\varepsilon$ (or complex conductivity $\sigma-i \omega \mathcal{E}$, or complex dielectric permittivity $\varepsilon+i \sigma / \omega)$, e.g., in [2-19]). According to the rules, the inverse problem is solved in frequency domain; that is, the external current source is harmonic. Despite the quite obvious idea that we can search for one complex function $\varepsilon+i \omega_{0} \sigma$, the authors assume numerical algorithms for reconstruction either of two real functions $\sigma$ and $\varepsilon$ or of real and imaginary parts of correspondent complex function, considering that one of the functions $\sigma$ or $\varepsilon$ is known. It leads to the fact that the resulting formulas are too complicated and inconvenient for analysis and implement on a computer. Apparently, another reason such numerical algorithms are offered is that when solving the inverse problem of finding a complex function $w$ by minimizing the functional $J[w]$, we go through the following steps: first, the functional $J: \mathbb{C} \rightarrow \mathbb{R}$ and, secondly, by the definition of the gradient, increment of the functional should be presented in the form $\delta J[w]=$ $\left\langle J^{\prime}[w], \delta w\right\rangle+o(\|\delta w\|)$; that is, there should be a scalar product for two complex values $\langle\cdot, \cdot\rangle: \mathbb{C} \times \mathbb{C} \rightarrow \mathbb{R}$.

In this paper the authors propose a numerical method of simultaneous definition of the conductivity $\sigma$ and dielectric permittivity $\varepsilon$ considering the example of inverse problem of subsurface radiolocation. Instead of two unknown real functions, we consider one complex. The authors generalize the known technique of finding of the gradient of the residual functional, using the statement of the conjugate problem for the case when the unknown function is complex valued. The notion of the reference frequency of the medium is introduced, which helps to understand where these two functions can be determined simultaneously. Some test reconstructions for simulated data are offered.

\section{Statement of the Inverse Problem}

We consider the media $-N_{l}$-layered structure with interfaces $z_{k}\left(k=\overline{0, N_{l}}\right), z_{0}=0 ; m$-layer is the interval $\left[z_{m-1}, z_{m}\right]$, the last $N_{l}+1$ (underlying) layer is the half space $\left[z_{N_{l}}, \infty\right.$ ), and the air is the half space $(-\infty, 0]$.

Electromagnetic properties of each layer are defined by the permittivity $\varepsilon_{0} \varepsilon$, the conductivity $\sigma$, and by magnetic 
permeability $\mu_{0} \mu, \varepsilon_{0}=8,854 \cdot 10^{-12} \mathrm{~F} / \mathrm{m}$ and $\mu_{0}=4 \pi$. $10^{-7} \mathrm{H} / \mathrm{m}$, in most cases the relative permittivity $\varepsilon$ belongs to the interval $[1 ; 80]$ and relative magnetic permeability $\mu=$ 1. Since the medium is horizontally stratified, then $\varepsilon$ and $\sigma$ are piecewise-constant functions of the variable $z(z \in$ $(-\infty, \infty))$.

Let the source of external current be a cable disposed on the height $z_{*}$ parallel to the $y$-axis.

For the component $E_{2}(t, x, z)$ from the Maxwell equations finally, we can obtain the differential equation of the second order (see, e.g., [1]):

$$
\varepsilon \frac{\partial^{2} E_{2}}{\partial t^{2}}+\sigma \frac{\partial E_{2}}{\partial t}=\frac{1}{\mu_{0}}\left(\frac{\partial^{2} E_{2}}{\partial x^{2}}+\frac{\partial^{2} E_{2}}{\partial z^{2}}\right) .
$$

The Fourier transform with respect to the horizontal variable $x$ and the time variable $t$ gives the following equation:

$$
u_{z z}-\left(\lambda^{2}-\omega^{2} \mu_{0} \varepsilon_{0} \varepsilon+i \omega \mu_{0} \sigma\right) u=0 .
$$

At points of discontinuity of the medium, we assume that the gluing conditions are as follows:

$$
[u]_{z_{k}}=0, \quad\left[u_{z}\right]_{z_{k}}=0, \quad k=\overline{0, N_{l}} \text {. }
$$

Source concentrated at the point $z_{*}=0$, which is equivalent to the gluing conditions at this point

$$
[u]_{z_{*}}=0, \quad\left[u_{z}\right]_{z_{*}}=-f(\omega) \mu_{0} .
$$

We assume that we have the conditions of damping in infinity

$$
u \longrightarrow 0 \quad(z \longrightarrow \pm \infty)
$$

and, relative to the solution of the direct problem (2)-(5), the additional information is given

$$
\left.u\right|_{z=0}=g(\omega, \lambda) .
$$

Here $\lambda$ and $\omega$ are the Fourier parameters with respect to variables $x$ and $t$, respectively, $[\cdot]_{z}$ is the notation for gluing, that is, $[w]_{z}=w(z+0)-w(z-0)$, and the bar over the complex value will denote the complex conjunction.

The inverse problem is to find the piecewise-constant functions $\varepsilon$ and $\sigma$, if for solution of the direct problem (2)(5), the additional information (6) is known.

Introduce the notation

$$
k^{2}=\lambda^{2}-\varkappa, \quad \varkappa=\omega^{2} \mu_{0} \varepsilon_{0} \varepsilon-i \omega \mu_{0} \sigma .
$$

Fix some values of the angular frequency $\omega_{0}$. In the inverse problem, we will recover the complex value $\varkappa_{0}\left(\varkappa_{0}=\right.$ $\left.\omega_{0}^{2} \mu_{0} \varepsilon_{0} \varepsilon-i \omega_{0} \mu_{0} \sigma\right)$ which is a piecewise-constant function since the functions $\varepsilon$ and $\sigma$ are piecewise constant.

As is easy to see, if we find $\varkappa_{0}$ as a solution of the inverse problems (2)-(6), we immediately recover the functions $\varepsilon$ and $\sigma$ as follows:

$$
\varepsilon=\frac{\operatorname{Re}\left[\varkappa_{0}\right]}{\omega_{0}^{2} \mu_{0} \varepsilon_{0}}, \quad \sigma=\frac{-\operatorname{Im}\left[\varkappa_{0}\right]}{\omega_{0} \mu_{0}} .
$$

Inverse problem (2)-(6) may be solved by minimizing the residual functionals as follows:

$$
J\left[\varkappa_{0}\right]=\sum_{\omega} h_{\omega}\left|u\left(0, \omega, \lambda_{0}\right)-g\left(\omega, \lambda_{0}\right)\right|^{2},
$$

(here $h_{\omega}$ are certain weight multipliers). Paying attention to that in the functional (9), we fix a value of the spatial frequency $\lambda_{0}$ and prepare additional information (6) for different values of the angular frequency $\omega$.

For minimization, we will use the gradient method, since the rate of convergence of such method is higher than that of the method that uses only the values of the functional; therefore, we need to get the gradient of the residual functional (9), which in turn requires the definition of the scalar multiplication of two complex numbers.

For two complex numbers $w_{1}$ and $w_{2}$, we introduce the following relation:

$$
\left\langle w_{1}, w_{2}\right\rangle=\frac{1}{2}\left(w_{1} \bar{w}_{2}+\bar{w}_{1} w_{2}\right)
$$

The relation (10) has all the properties of scalar multiplication. The proof of this is based on a geometric interpretation of complex numbers. Let $w_{1}=\left(\alpha_{1}, \beta_{1}\right)$ and $w_{2}=\left(\alpha_{2}, \beta_{2}\right)$; then, $\left\langle w_{1}, w_{2}\right\rangle=\alpha_{1} \alpha_{2}+\beta_{1} \beta_{2}$.

For (10) note useful relation

$$
\left\langle w_{1}, w_{2}\right\rangle=\operatorname{Re}\left[w_{1} \bar{w}_{2}\right]=\operatorname{Re}\left[\bar{w}_{1} w_{2}\right]
$$

\section{Gradient of the Residual Functional}

First we note that

$$
J\left[\varkappa_{0}\right]=J\left[\varkappa_{0}^{1}, \ldots, \varkappa_{0}^{m}, \ldots, \varkappa_{0}^{N_{l}}\right],
$$

where $\varkappa_{0}^{m}$ are the values of a piecewise constant function $\varkappa_{0}$ in the segment $\left[z_{m-1}, z_{m}\right]$.

We obtain the expression of the gradient of the residual functional (9) by the statement of the conjugate problem

$$
\begin{gathered}
\psi_{z z}-\left(\lambda^{2}-\varkappa\right) \psi=0 \\
{[\psi]_{z_{k}}=0, \quad\left[\psi_{z}\right]_{z_{k}}=0, \quad k=\overline{1, N_{l}},} \\
{[\psi]_{z_{*}}=0, \quad\left[\psi_{z}\right]_{z_{*}}=-2\left[\overline{u\left(0, \omega_{0}, \lambda\right)}-\overline{g\left(\omega_{0}\right)}\right],} \\
\psi \rightarrow 0 \quad(z \longrightarrow \pm \infty) .
\end{gathered}
$$

Let the value $\varkappa$ be incremented $\delta \varkappa$; then, the function $u$ will get increment $\delta u$, which satisfies the following problem:

$$
\begin{gathered}
\delta u_{z z}-k^{2} \delta u+u \delta \varkappa=0, \\
{[\delta u]_{z_{k}}=0, \quad\left[\delta u_{z}\right]_{z_{k}}=0, \quad k=\overline{-1, N_{l}},} \\
u \longrightarrow 0 \quad(z \longrightarrow \pm \infty)
\end{gathered}
$$

(here for, simplicity, we set $z_{-1}=z_{*}$ ). 
In this case, the increment of the residual functional up the second order can be obtained as follows:

$$
\delta J\left[\varkappa_{0}\right]=\operatorname{Re}\left[\sum_{\lambda} 2 h_{\lambda}(\bar{u}-\bar{g}) \delta u\right]=-\operatorname{Re}\left(\sum_{\lambda} h_{\lambda}[\psi]_{z_{0}} \delta u\right) .
$$

Given statements of problems (14), (13), and the equality

$$
\int_{-\infty}^{\infty} \frac{d}{d z}\left(\delta u \psi_{z}\right) d z=-\left.\delta u\right|_{z=z_{0}}\left[\psi_{z}\right]_{z_{0}},
$$

we get

$$
\delta J\left[\varkappa_{0}\right]=\operatorname{Re}\left[\sum_{\lambda} h_{\lambda} \int_{z_{0}}^{z_{N_{l}}} u \psi \delta \varkappa d x\right] .
$$

Since in each layer $\left[z_{m-1}, z_{m}\right]$, the function $\varkappa$ is constant and takes the value $\varkappa^{m}$, we can write

$$
\delta J\left[\varkappa_{0}\right]=\sum_{m=1}^{N_{l}} \operatorname{Re}\left[\sum_{\omega} h_{\omega} \int_{z_{m-1}}^{z_{m}} u \psi d z \cdot \delta \varkappa^{m}\right] .
$$

Let

$$
N_{m}=\int_{z_{m-1}}^{z_{m}} u \psi d z
$$

Then

$$
\begin{aligned}
\operatorname{Re}\left[N_{m} \delta \varkappa^{m}\right]= & \operatorname{Re}\left[N_{m}\right] \cdot \omega^{2} \mu_{0} \varepsilon_{0} \delta \varepsilon_{m}+\operatorname{Im}\left[N_{m}\right] \cdot \omega \mu_{0} \delta \sigma_{m} \\
= & \operatorname{Re}\left[N_{m}\right] \cdot \frac{\omega^{2}}{\omega_{0}^{2}} \cdot \omega_{0}^{2} \mu_{0} \varepsilon_{0} \delta \varepsilon_{m} \\
& +\operatorname{Im}\left[N_{m}\right] \cdot \frac{\omega}{\omega_{0}} \cdot \omega_{0} \mu_{0} \delta \sigma_{m} \\
= & \operatorname{Re}\left[\widehat{N}_{m} \delta \varkappa_{0}^{m}\right],
\end{aligned}
$$

where

$$
\widehat{N}_{m}=\operatorname{Re}\left[N_{m}\right] \frac{\omega^{2}}{\omega_{0}^{2}}+i \operatorname{Im}\left[N_{m}\right] \frac{\omega}{\omega_{0}} .
$$

Therefore, the gradient of the residual functional $J\left[\varkappa_{0}\right]$ will have the form

$$
J^{\prime}\left[\varkappa_{0}\right]=\left(\bar{j}_{1}, \ldots, \bar{j}_{m}, \ldots, \bar{j}_{N_{l}}\right), \quad j_{m}=\sum_{\omega} h_{\omega} \widehat{N}_{m} .
$$

\section{Analytic Formulas for the Key Expressions}

In order to solve the direct problem (2)-(5) and conjugate problem (13), we will reduce the differential equation of the second order to the Riccati equation. This method was successfully used, for example, in [20-23].

For solving differential equation (2), we introduce the function $s$ as follows:

$$
u_{z}(z)=s(z) u(z),
$$

which will satisfy the differential Riccati equation

$$
s^{\prime}+s^{2}=k^{2} .
$$

If we calculate from the right to the left, then the solution of (24) in each segment $\left[z_{m-1}, z_{m}\right]$ will be as follows:

$$
s(z)=k_{m} \frac{\left(s^{m}+k_{m}\right) e^{2 k_{m}\left(z-z_{m}\right)}+\left(s^{m}-k_{m}\right)}{\left(s^{m}+k_{m}\right) e^{2 k_{m}\left(z-z_{m}\right)}-\left(s^{m}-k_{m}\right)} .
$$

If we calculate from the left to the right, then

$$
s(z)=k_{m} \frac{\left(s^{m-1}+k_{m}\right)+\left(s^{m-1}-k_{m}\right) e^{2 k_{m}\left(z_{m-1}-z\right)}}{\left(s^{m-1}+k_{m}\right)-\left(s^{m-1}-k_{m}\right) e^{2 k_{m}\left(z_{m-1}-z\right)}}
$$

(here $s^{m}=\left.s\right|_{z=z_{m}}, k_{m}$ is the value of the piecewise constant function $k$ in the segment $\left[z_{m-1}, z_{m}\right]$ and $\operatorname{Re}\left[k_{m}\right] \geq 0$ ).

For solving Riccati equation (24), we will do our recurrent calculations from the layer up to the layer moving to the point $z_{*}$, where the source is disposed.

The condition of damping in infinity (5) enables

$$
s(z)=-k_{N_{l}+1}, \quad z \in\left[z_{N_{l}}, \infty\right) .
$$

Due to conditions (6), we obtain the gluing conditions

$$
[s]_{z_{k}}=0 .
$$

Therefore, we may set

$$
s^{N_{l}}=-k_{N_{l}+1}
$$

and begin the recurrent calculation of $s^{m}$ from the right to the left by formula

$$
\begin{array}{r}
s^{m-1}=k_{m} \frac{\left(s^{m}+k_{m}\right) e^{-2 k_{m} h_{m}}+\left(s^{m}-k_{m}\right)}{\left(s^{m}+k_{m}\right) e^{-2 k_{m} h_{m}}-\left(s^{m}-k_{m}\right)}, \\
h_{m}=z_{m}-z_{m-1} .
\end{array}
$$

Thus we get $s^{*}=\left.s\right|_{z=z_{*}+0}$.

Analogously, taking into account damping in minus infinity (5) we get

$$
s(z)=k_{0}, \quad z \in\left(\infty, z_{*}\right] .
$$

Since the source is disposed in the half space $(-\infty, 0]$, we may immediately take

$$
\left.s\right|_{z=z_{*}-0}=k_{0} .
$$

Gluing condition (28) in the point $z=z_{*}$ allows us to determine

$$
\left.u\right|_{z=z_{*}} \equiv u^{*}=-\frac{f(\omega) \mu_{0}}{s^{*}-k_{0}} .
$$

Integrating (23) in the interval $\left[z_{*}, 0\right]$, we obtain

$$
\left.u\right|_{z=0} \equiv u^{0}=u^{*} \frac{2 k_{0} e^{-k_{0} z_{*}}}{\left(s^{0}+k_{0}\right) e^{-2 k_{0} z_{*}}-\left(s^{0}-k_{0}\right)} .
$$


Further, integrating in each interval $\left[z_{m-1}, z_{m}\right]$ (23), we derive the solution of the problem (2)-(5) in this interval

$$
\begin{gathered}
u(z)=u^{m-1} l(z), \\
l(z)=e^{k_{m}\left(z_{m-1}-z\right)} \frac{\left(s^{m}+k_{m}\right) e^{2 k_{m}\left(z-z_{m}\right)}-\left(s^{m}-k_{m}\right)}{\left(s^{m}+k_{m}\right) e^{-2 k_{m} h_{m}}-\left(s^{m}-k_{m}\right)} .
\end{gathered}
$$

The conjugate problem (13) is similar to direct problem (2)-(5); therefore, it can be solved similarly. Moreover, since the damping conditions in infinity are the same, the solution of the Riccati equation introduced for the function $\psi$ will coincide with $s(z)$ for all $z \in[0, \infty)$. Therefore, we have

$$
\left.\psi\right|_{z=0} \equiv \psi^{0}=-\frac{2[\overline{u(0, \omega, \lambda)}-\overline{g(\omega, \lambda)}]}{s^{0}-k_{0}}
$$

and in each interval $\left[z_{m-1}, z_{m}\right]$.

$$
\psi(z)=\psi^{m-1} l(z) \text {. }
$$

Taking into account (35) and (37), we can obtain the formulas for the gradient components of $J_{\omega}\left[\varkappa_{0}\right](\alpha=\lambda, \omega)$; that is, calculate the integral

$$
\int_{z_{m-1}}^{z_{m}} u \psi d z=\frac{u^{m-1} \psi^{m-1} R^{m}}{\left[\left(s^{m}+k_{m}\right) e^{-2 k_{m} h_{m}}-\left(s^{m}-k_{m}\right)\right]^{2}},
$$

where

$$
\begin{aligned}
R^{m}= & \frac{1-e^{-2 k_{m} h_{m}}}{2 k_{m}} \\
& \times\left[\left(s^{m}+k_{m}\right)^{2} e^{-2 k_{m} h_{m}}+\left(s^{m}-k_{m}\right)^{2}\right] \\
& -2 h_{m}\left(s^{m}+k_{m}\right)\left(s^{m}-k_{m}\right) e^{-2 k_{m} h_{m}} .
\end{aligned}
$$

\section{Numerical Experiment}

5.1. Reference Frequency of the Medium $\omega_{0}$. First we note, that (see, e.g., formulas (25) and (35))

$$
u=u(k)
$$

where

$$
\begin{gathered}
k^{2}=\lambda^{2}-\left(\omega^{2} \mu_{0} \varepsilon_{0} \varepsilon-i \omega \mu_{0} \sigma\right), \\
k=\sqrt[4]{\left[\lambda^{2}-\omega^{2} \mu_{0} \varepsilon_{0} \varepsilon\right]^{2}+\left[\omega \mu_{0} \sigma\right]^{2}} e^{i \beta / 2}, \\
\beta= \begin{cases}\operatorname{arctg} \frac{\omega \mu_{0} \sigma}{\lambda^{2}-\omega^{2} \mu_{0} \varepsilon_{0} \varepsilon}, & \lambda^{2}>\omega^{2} \mu_{0} \varepsilon_{0} \varepsilon \\
\frac{\pi}{2}, & \lambda^{2}=\omega^{2} \mu_{0} \varepsilon_{0} \varepsilon \\
\pi+\operatorname{arctg} \frac{\omega \mu_{0} \sigma}{\lambda^{2}-\omega^{2} \mu_{0} \varepsilon_{0} \varepsilon} & \lambda^{2}<\omega^{2} \mu_{0} \varepsilon_{0} \varepsilon\end{cases}
\end{gathered}
$$

That is, the variation of the solution of direct problem (2)-(5) depends directly on how the function $k$ depends on variations of the functions $\varepsilon$ and $\sigma$ in the layers.
TABLE 1: Model of the medium 1.

\begin{tabular}{lcccccc}
\hline Number of the layer & 1 & 2 & 3 & 4 & 5 & 6 \\
\hline$\varepsilon$ & 18.5 & 22.8 & 18.4 & 19.2 & 28.3 & 30.0 \\
$\sigma$ & 0.017 & 0.024 & 0.016 & 0.017 & 0.022 & 0.024 \\
$z_{k}$ & 0.11 & 0.21 & 0.39 & 0.58 & 0.82 & \\
\hline
\end{tabular}

TABLE 2: Model of the medium 2.

\begin{tabular}{lcccccc}
\hline Number of the layer & 1 & 2 & 3 & 4 & 5 & 6 \\
\hline$\varepsilon$ & 20.5 & 22.7 & 17.9 & 22.8 & 21.0 & 30.0 \\
$\sigma$ & 0.021 & 0.023 & 0.020 & 0.021 & 0.020 & 0.025 \\
$z_{k}$ & 0.11 & 0.22 & 0.39 & 0.62 & 0.82 & \\
\hline
\end{tabular}

TABLe 3: Model of the medium 3.

\begin{tabular}{lcccccc}
\hline $\begin{array}{l}\text { Number of } \\
\text { the layer }\end{array}$ & 1 & 2 & 3 & 4 & 5 & 6 \\
\hline$\varepsilon$ & 2.05 & 2.27 & 2.05 & 2.08 & 1.93 & 2.50 \\
$\sigma$ & 0.0021 & 0.0023 & 0.0020 & 0.0021 & 0.0020 & 0.0025 \\
$z_{k}$ & 0.48 & 0.88 & 1.38 & 2.00 & 2.50 & \\
\hline
\end{tabular}

Evidently, the greatest influence on change in the value $k$ is rendered by variations $\varepsilon$ and $\sigma$, when

$$
\omega^{2} \mu_{0} \varepsilon_{0} \varepsilon \approx \omega \mu_{0} \sigma, \quad \lambda^{2} \sim \omega^{2} \mu_{0} \varepsilon_{0} \varepsilon .
$$

We put $\omega^{2} \mu_{0} \varepsilon_{0} \varepsilon=\omega \mu_{0} \sigma$, whence we get

$$
\omega_{0}=\frac{\sigma}{\varepsilon_{0} \varepsilon} .
$$

This means that we know for certain medium the mean values of dielectric permittivity and conductivity. Then we derive and fix the value of reference frequency of the medium (43).

5.2. Dependence of the Properties of the Functional $J[\varkappa]$ from the Value $\omega$. For numerical experiments below we choose model shown in Table 1.

We set the mean values at $\varepsilon=20$ and $\sigma=0,020$, and then the reference angular frequency is $\omega_{0}=1.12 \cdot 10^{8}$. The capacity of the skin-layer, from here, is

$$
h_{s}=\sqrt{\frac{2}{\omega_{0} \mu_{0} \sigma}} \approx 0.84(\mathrm{~m}) \text {. }
$$

Fixing the values $\varepsilon_{m}$ and $\sigma_{m}(m=\overline{2,5})$, we will change the values of dielectric permittivity and conductivity of the first layer in the segment

$$
\varepsilon_{1} \in[1.0,40.0], \quad \sigma_{1} \in[0.01,0.03],
$$

for different values of $\omega=r \omega_{0}$, where $r=\{1 / 10,1 / 7,1 / 5,1 / 2$, $1,2,5,7,10\}$ and we will observe the behavior of the value

$$
\Delta_{\omega}=\left|u\left(0, \omega, \lambda_{0}\right)-g\left(\omega, \lambda_{0}\right)\right|^{2},
$$

which is one of the components of the residual functional (9). Results of numerical experiment are presented in Figure 1. 

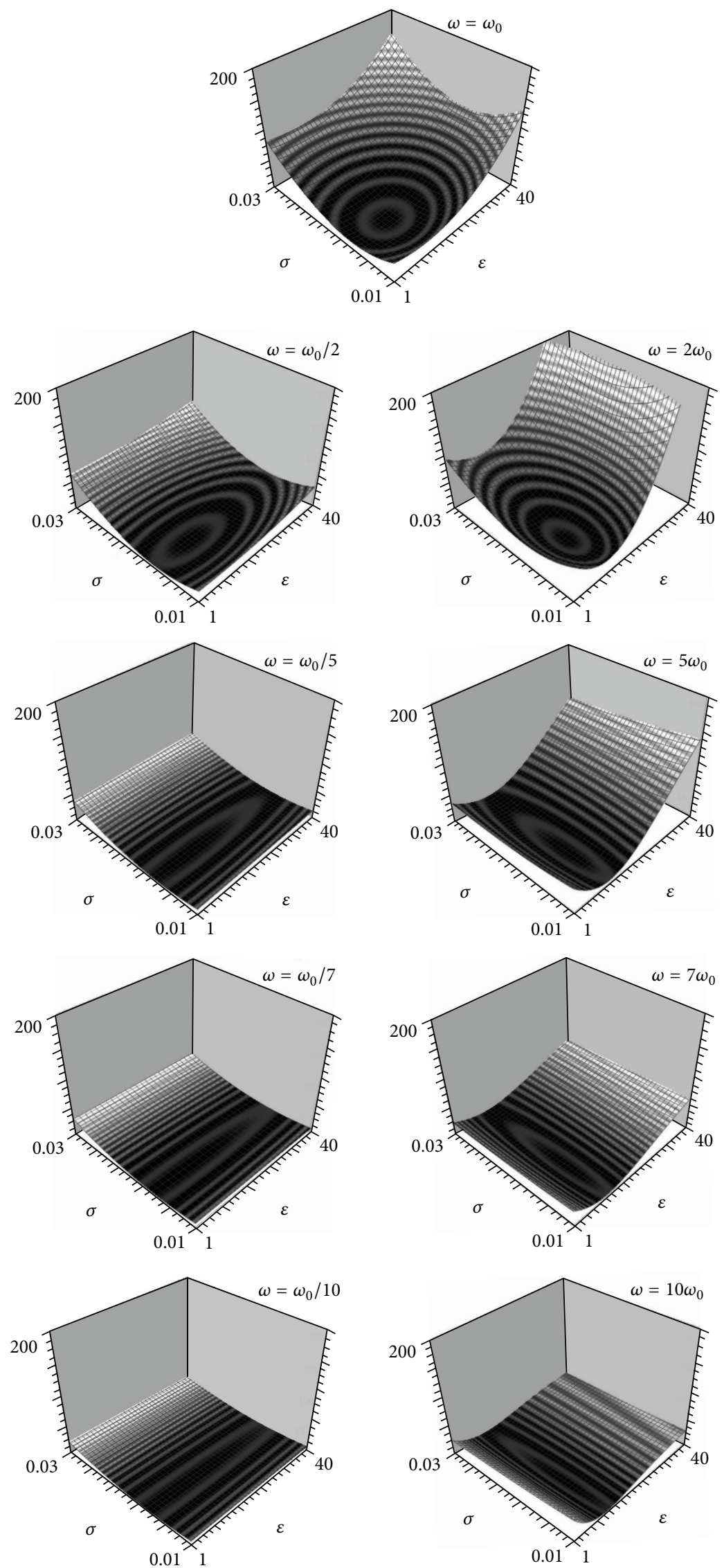

FIgURE 1: The behavior of $\Delta_{\omega}$ for various values $\omega$. 
TABLe 4: Model of the medium 4.

\begin{tabular}{lccccccccccc}
\hline Number of the layer & 1 & 2 & 3 & 4 & 5 & 6 & 7 & 8 & 9 & 10 \\
\hline$\varepsilon$ & 20.2 & 21.3 & 22.1 & 20.4 & 18.4 & 16.2 & 17.8 & 18.8 & 22.2 & 23.3 & 25.0 \\
$\sigma$ & 0.0018 & 0.0021 & 0.0020 & 0.0021 & 0.0020 & 0.0018 & 0.0019 & 0.0021 & 0.0022 & 0.0023 & 0.0025 \\
$z_{k}$ & 0.72 & 1.53 & 2.91 & 3.65 & 4.45 & 5.05 & 6.00 & 6.65 & 7.23 & 8.20 \\
\hline
\end{tabular}

TABle 5: Model of the medium 5.

\begin{tabular}{lcccccccc}
\hline Number of the layer & 1 & 2 & 3 & 4 & 5 & 6 & 7 & 8 \\
\hline$\varepsilon$ & 20.2 & 19.3 & 20.2 & 20.9 & 21.1 & 19.2 & 23.4 & 2.50 \\
$\sigma$ & 0.021 & 0.020 & 0.021 & 0.019 & 0.018 & 0.019 & 0.022 & 0.025 \\
$z_{k}$ & 0.11 & 0.22 & 0.33 & 0.44 & 0.59 & 0.72 & 0.83 \\
\hline
\end{tabular}

TABLE 6

\begin{tabular}{|c|c|c|c|}
\hline & $\omega_{0}$ & Frequency interval & $\begin{array}{l}\text { Number of } \\
\text { frequencies }\end{array}$ \\
\hline $\begin{array}{l}\text { Model of the } \\
\text { medium } 1\end{array}$ & $1.12 \cdot 10^{8}$ & {$\left[\omega_{0} / 10,10 \omega_{0}\right]$} & 2500 \\
\hline $\begin{array}{l}\text { Model of the } \\
\text { medium } 2\end{array}$ & $1.12 \cdot 10^{8}$ & {$\left[\omega_{0} / 10,10 \omega_{0}\right]$} & 2500 \\
\hline $\begin{array}{l}\text { Model of the } \\
\text { medium } 3\end{array}$ & $1.12 \cdot 10^{8}$ & {$\left[\omega_{0} / 10,10 \omega_{0}\right]$} & 2500 \\
\hline $\begin{array}{l}\text { Model of the } \\
\text { medium } 4\end{array}$ & $1.12 \cdot 10^{7}$ & {$\left[\omega_{0} / 40,40 \omega_{0}\right]$} & 6000 \\
\hline $\begin{array}{l}\text { Model of the } \\
\text { medium } 5\end{array}$ & $1.12 \cdot 10^{8}$ & {$\left[\omega_{0} / 40,40 \omega_{0}\right]$} & 6000 \\
\hline
\end{tabular}

We note that first, if $\omega$ decreases with respect to the reference frequency of the medium $\omega_{0}$, then the residual functional loses sensitivity when $\varepsilon_{1}$ varies. If $\omega$ increases, then the functional loses the sensitivity when $\sigma_{1}$ varies. Secondly, the more the difference between $\omega$ and $\omega_{0}$ the less the sensitivity of the residual functional $J[\varkappa]$ to the change of desired unknown values, since it becomes more standing.

If $\varepsilon_{m}$ and $\sigma_{m}(m=\overline{2,5})$ vary, the behavior of $\Delta_{\omega}$ will be similar; however, if the layer is lower, so is the sensitivity of the functional.

Thus, the assumption that the value of the angular frequency $\omega$ must by such that

$$
\omega^{2} \mu_{0} \varepsilon_{0} \varepsilon \approx \omega \mu_{0} \sigma
$$

is verified (see (42)).

In addition, we clarify the known geophysical condition of quasistationarity of the electromagnetic field: $\omega \varepsilon_{0} \varepsilon \ll \sigma$. The value of notation “«” is lax. Proceeding from the numerical experiment, we may account the quasistationary approximation to be suitable if $\omega \varepsilon_{0} \varepsilon \leq \sigma / 10$.

5.3. Dependence of the Properties of the Residual Functional from the Value $\lambda$. For the model 1 (see Table 1), the numerical experiment similar to those in the previous section was developed. The values of the dielectric permittivity and conductivity of the first layer were changing (in above mentioned segments) and the values of the residual functional $J\left[\varkappa_{0}\right]$ were derived. The result is shown in Figure 2.

The tendency is seen as the greater $\lambda$ the less is the sensitivity of residual functional to variations of the unknown parameters. The assumption that

$$
\lambda^{2} \sim \omega^{2} \mu_{0} \varepsilon_{0} \varepsilon
$$

is verified.

For model 1 we obtain $\omega^{2} \mu_{0} \varepsilon_{0} \varepsilon \approx 2.84$. It is seen that with increasing $\lambda$ at first the residual functional $J\left[\varkappa_{0}\right]$ loses the sensitivity to the variations of $\sigma_{1}$ and then to variations of $\varepsilon_{1}$. When $\lambda^{2} \geq 2 \omega^{2} \mu_{0} \varepsilon_{0} \varepsilon$, the sensitivity of the residual functional rapidly decreases.

\section{Numerical Examples of Solution of the Inverse Problem}

In order to test the operability of the proposed numerical algorithm, we carry out a number of reconstruction of electromagnetic properties of the medium, using simulated data. In order to obtain the additional information (6) we, first, solve the direct problem (2)-(5). Then we add the random value with this form

$$
\tilde{g}\left(\omega, \lambda_{0}\right)=g\left(\omega, \lambda_{0}\right)\left(1+\frac{P}{100} \xi\right),
$$

where $\xi$ is a random value from the unit circle and $P$ is the percent of the introduced error. In Figure 3 we see the example of using the additional information with this error.

In addition to the model of the medium 1 in Table 1 were selected four more models (see Tables 2, 3, 4, and 5).

For each model of the medium the coordinate of the boundary of the last layer $z_{N_{l}}$ coincides with the value of capacity of the skin-layer $h_{s}$.

The parameters used in the construction of the residual functional $J\left[\varkappa_{0}\right]$ are collected in Table 6 .

In each layer, the initial approximations were $\varepsilon_{N_{l}}$ and $\sigma_{N_{l}}$. 

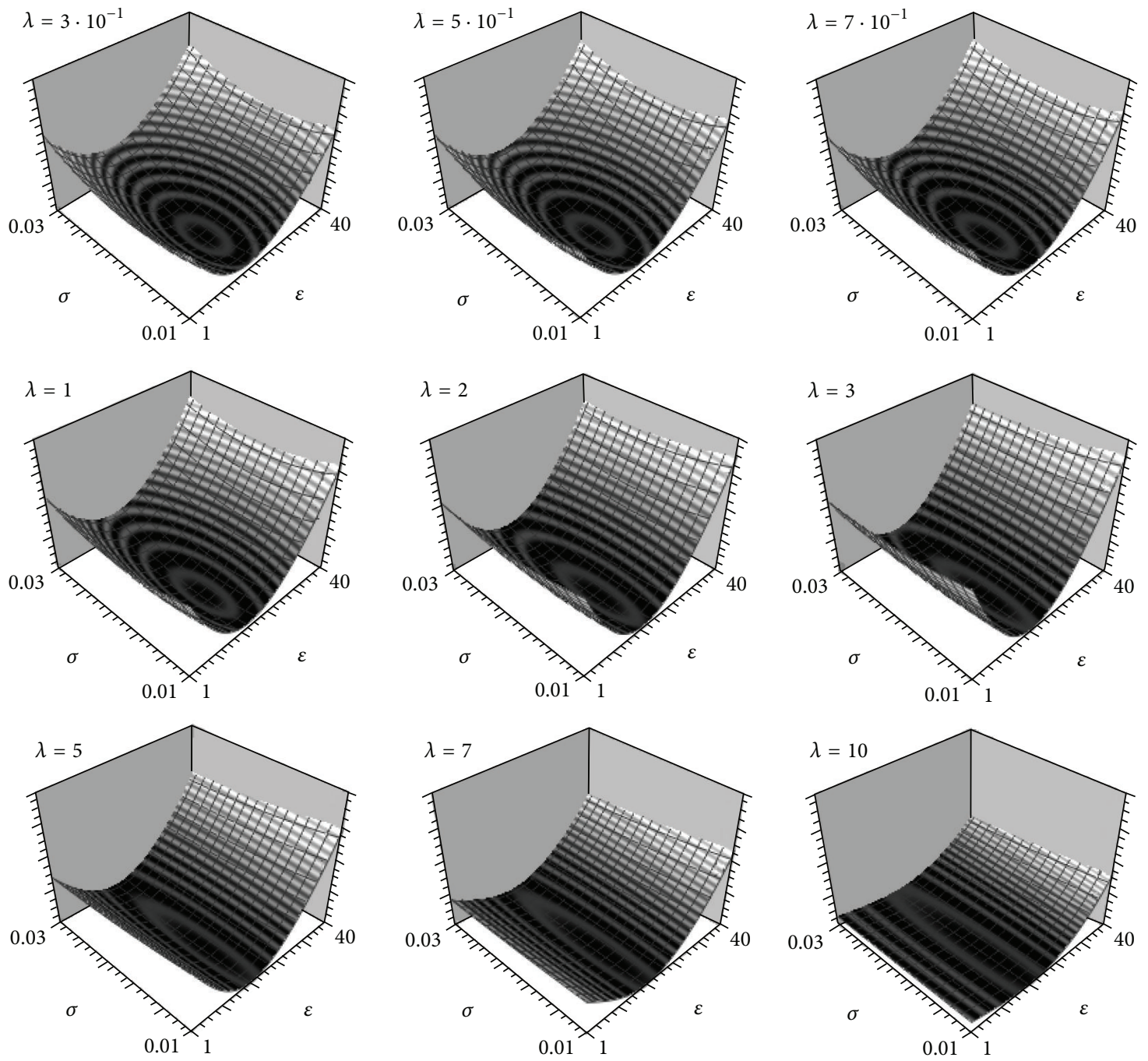

Figure 2: The behavior of the residual functional $J\left[\varkappa_{0}\right]$ for various values of $\lambda .450$ values of $\omega$ were taken from the segment $\left[\omega_{0} / 10 ; 10 \omega_{0}\right]$ with constant step.

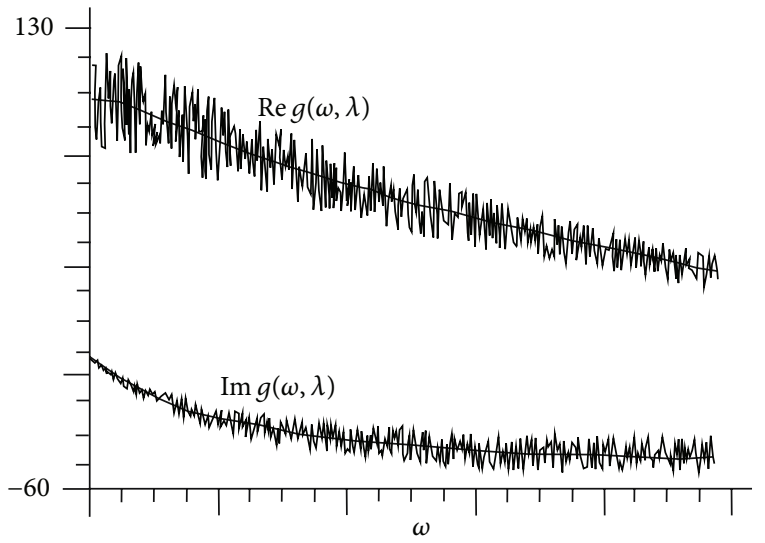

FIGURE 3: The example of additional information $g(\omega, \lambda)$ given with the error $P=20 \%\left(\omega \in\left[\omega_{0} / 50, \omega_{0} / 10\right], \lambda=5 \cdot 10^{-1}\right)$; the exact values of real and imaginary parts of $g(\omega, \lambda)$ are shown by the smooth line.
For minimizing of the residual functional $J\left[\varkappa_{0}\right]$ the conjugate gradient method modified for complex values used

$$
\begin{gathered}
\varkappa_{0}^{n+1}=\varkappa_{0}^{n}-\alpha_{n} p_{n}, \\
p_{n}=J^{\prime}\left[\varkappa_{0}^{n}\right]-\beta_{n} p_{n-1}, \quad p_{0}=J^{\prime}\left[\varkappa_{0}^{0}\right], \\
\beta_{n}=-\frac{\left\|J^{\prime}\left[\varkappa_{0}^{n}\right]\right\|^{2}}{\left\|J^{\prime}\left[\varkappa_{0}^{n-1}\right]\right\|^{2}} \\
\alpha_{n}=\arg \min _{\alpha>0} J\left[\varkappa_{0}^{n}-\alpha p_{n}\right] .
\end{gathered}
$$

The results of restoration of piecewise constant functions $\varepsilon$ and $\sigma$ are shown in Figure 4 .

Remember that we used the value of the reference angular frequency $\omega_{0}$ such that the condition $\omega_{0}^{2} \mu_{0} \varepsilon_{0} \varepsilon \approx \omega_{0} \mu_{0} \sigma$ is satisfied. Recovery experiment shows that the less the value 

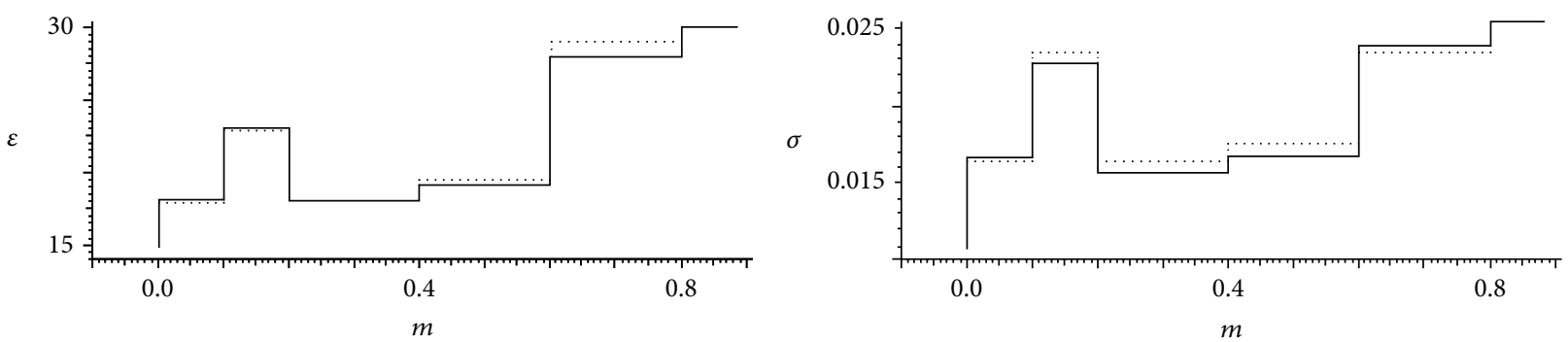

(a)
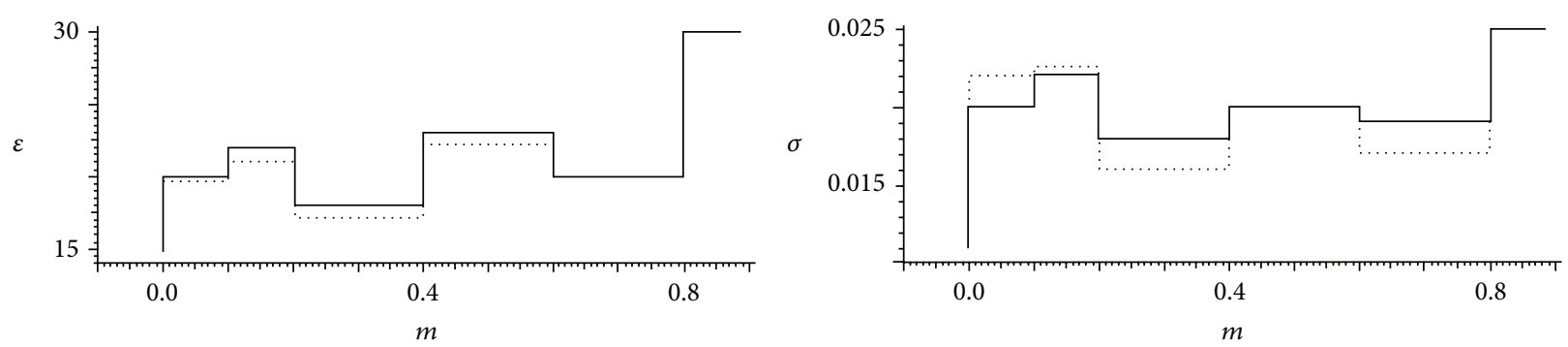

(b)
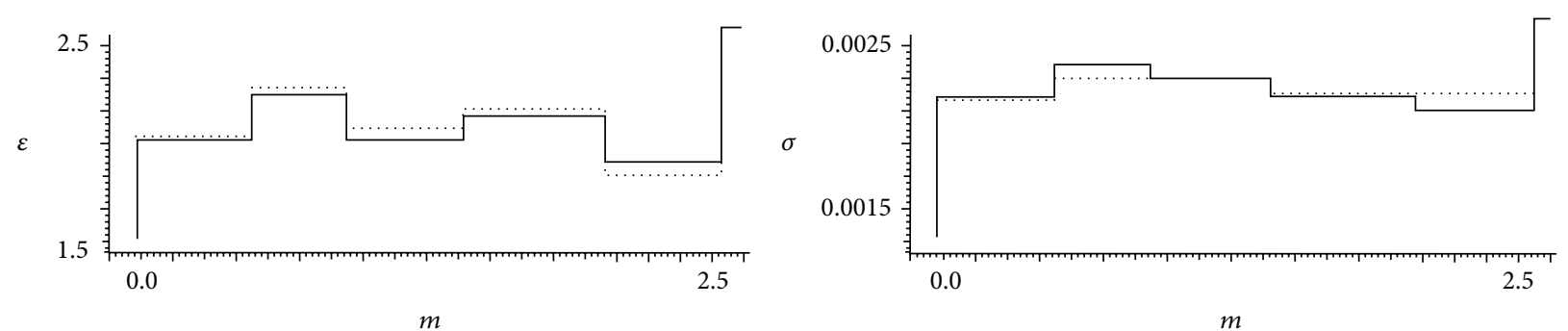

(c)
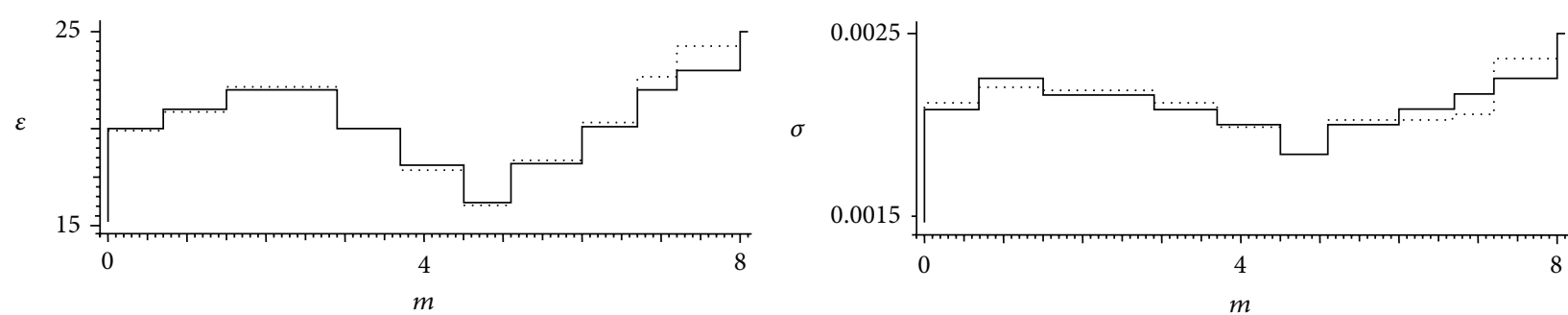

(d)
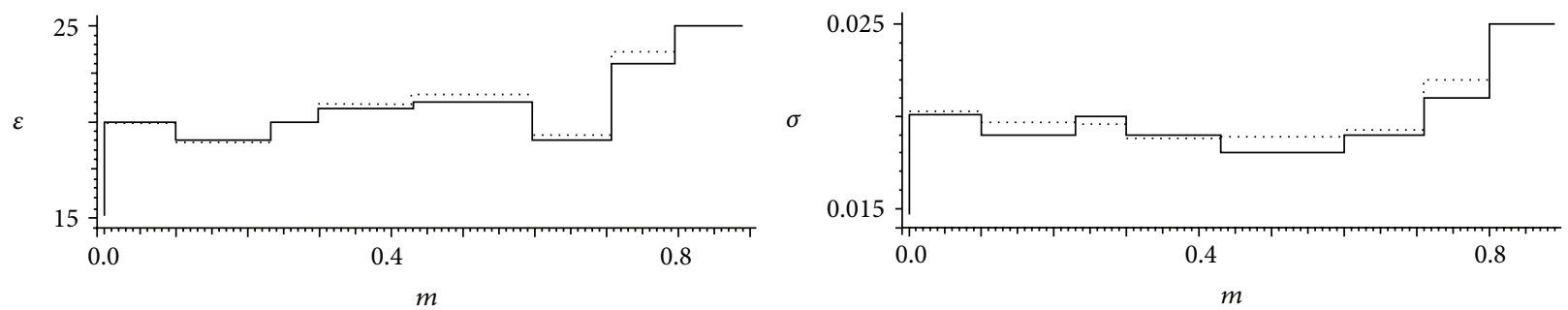

(e)

FIGURE 4: The examples or recovery $\varepsilon$ and $\sigma$ : (a) model of the medium 1, (b) model of the medium 2, (c) model of the medium 3, (d) model of the medium 4, and (e) model of the medium 5 . The exact solution is shown by the continuous line. The recovered solution is shown by the dotted line. For all the examples $P=20 \%$. 
of $\omega \mu_{0} \sigma$, the more difficult restoring of the functions $\varepsilon$ and $\sigma$ is, since small values of $k^{2}$ in the differential equation (2) render little impact on changing values of the solution of the equation. The consequence of this is the large "flatness" of the residual functional and its low sensitivity to variations of $\varepsilon$ and $\sigma$. Note: $\omega_{0} \mu_{0} \sigma=2 / h_{s}^{2}$.

\section{Conclusions}

In this paper, the authors suggested the generalization of the known technique of constructing the gradient of the residual functional with the use of the statement of the conjugate problem, when the unknown function is complex.

The numerical examples had shown that the conjugate gradient method in the case of complex valued gradient of the residual functional and the known function is applicable and we can find the minimum of the functional.

Efficiency rate of the method is confirmed by examples of simultaneous reconstruction of the dielectric permittivity and conductivity on synthetic data with introduced random error.

\section{Acknowledgments}

The work was supported by the project of Ministry of Education and Science of the Republic of Kazakhstan (Grant 1173/GF2 (N. 378 from 04.02.2013)), Grant N. 773 from 01.10.2013, SB RAS and NAS of Ukraine (Project 12-2013), and RFBR (Grant 12-01-00773).

\section{References}

[1] V. G. Romanov and S. I. Kabanikhin, Inverse Problems for Maxwell's Equations, VSP, Utrecht, The Netherlands, 1994.

[2] F. Ahmad and M. Razzaghi, "Simultaneous reconstruction of approximate profiles of an inhomogeneous lossy medium through a collocation method," Journal of Physics D, vol. 30, no. 23, pp. 3274-3278, 1997.

[3] L. Crocco and F. Soldovieri, "GPR prospecting in a layered medium via microwave tomography," Annals of Geophysics, vol. 46, no. 3, pp. 559-572, 2003.

[4] D. G. Drogoudis, G. A. Kyriacou, and J. N. Sahalos, "A sensitivity matrix based microwave tomography exploiting an adjoint network theorem," in Proceedings of the Progress in Electromagnetics Research Symposium (PIERS '07), pp. 272-276, Prague, Czech Republic, August 2007.

[5] O. Dorn, E. L. Miller, and C. M. Rappaport, "A shape reconstruction method for electromagnetic tomography using adjoint fields and level sets," Inverse Problems, vol. 16, no. 5, pp. 11191156, 2000.

[6] E. E. Eves, E. K. Murphy, and V. V. Yakovlev, "Practical aspects of complex permittivity reconstruction with neural-networkcontrolled FDTD modeling of a two-port fixture," Journal of Microwave Power and Electromagnetic Energy, vol. 41, no. 4, pp. 81-94, 2007.

[7] C. G. Farquharson, D. W. Oldenburg, and P. S. Routh, "Simultaneous 1D inversion of loop-loop electromagnetic data for magnetic susceptibility and electrical conductivity," Geophysics, vol. 68, no. 6, pp. 1857-1869, 2003.

[8] T. M. Habashy, W. C. Chew, and E. Y. Chow, "Simultaneous reconstruction of permittivity and conductivity profiles in a radially inhomogeneous slab," Radio Science, vol. 21, no. 4, pp. 635-645, 1986.

[9] T. M. Habashy, M. L. Oristaglio, and A. T. de Hoop, "Simultaneous nonlinear reconstruction of two-dimensional permittivity and conductivity," Radio Science, vol. 29, no. 4, pp. 1101-1118, 1994.

[10] K. Hollaus, C. Gerstenberger, Ch. Magele, and H. Hutten, "Accurate reconstruction algorithm of the complex conductivity distribution in three dimensions," IEEE Transactions on Magnetics, vol. 40, no. 2, pp. 1144-1147, 2004.

[11] H. Jain, D. Isaacson, P. M. Edic, and J. C. Newell, "Electrical impedance tomography of complex conductivity distributions with noncircular boundary," IEEE Transactions on Biomedical Engineering, vol. 44, no. 11, pp. 1051-1060, 1997.

[12] C. N. Kechribaris, K. S. Nikita, and N. K. Uzunoglu, "Reconstruction of two-dimensional permittivity distribution using an improved Rytov approximation and nonlinear optimization," Journal of Electromagnetic Waves and Applications, vol. 17, no. 2, pp. 183-207, 2003.

[13] W. R. B. Lionheart, S. R. Arridge, M. Schweiger, M. Vauhkonen, and J. P. Kaipio, "Electrical impedance and diffuse optical tomography reconstruction software," in Proceedings of $1 s t$ World Congress on Industrial Process Tomography, pp. 474-477, Buxton, UK, April 1999.

[14] T. T. Rekanos and Th. D. Tsiboukis, "A combined finite element-nonlinear conjugate gradient spatial method for the reconstruction of unknown scatterer profiles," IEEE Transactions on Magnetics, vol. 34, no. 5, pp. 2829-2832, 1998.

[15] L. T. Rekanos, S. M. Panas, and Th. D. Tsiboukis, "Microwave imaging using the finite-element method and a sensitivity analysis approach," IEEE Transactions on Medical Imaging, vol. 18, no. 11, pp. 1108-1114, 1999.

[16] A. G. Sena and M. N. Toksoz, "Simultaneous reconstruction of permittivity and conductivity for crosshole geometries," Geophysics, vol. 55, no. 10, pp. 1302-1311, 1990.

[17] M. Soleimani, W. R. B. Lionheart, and O. Dorn, "Level set reconstruction of conductivity and permittivity from boundary electrical measurements using experimental data," Inverse Problems in Science and Engineering, vol. 14, no. 2, pp. 193-210, 2006.

[18] B. Wagner, K. Hollaus, Ch. Magele, and R. Stollberger, "Reconstruction of the anisotropic complex conductivity distribution of a human thigh in three dimensions," IEEE Transactions on Magnetics, vol. 42, no. 4, pp. 1171-1174, 2006.

[19] Zh. Zhang and D. W. Oldenburg, "Simultaneous reconstruction of 1-D susceptibility and conductivity from electromagnetic data," Geophysics, vol. 64, no. 1, pp. 33-47, 1999.

[20] V. I. Dmitriev, "General method for calculating the electromagnetic field in the layered medium," Numerical Methods and Programming, vol. 10, pp. 55-65, 1968.

[21] V. I. Dmitriev and E. A. Fedorova, "Numerical study of electromagnetic fields in the layered medium," Numerical Methods and Programming, vol. 32, pp. 150-183, 1980.

[22] A. L. Karchevsky, "Numerical solution to system of elasticity equations for layered anisotropic media," Russian Geology and Geophysics, vol. 46, no. 3, pp. 339-351, 2005.

[23] A. L. Karchevsky, "Direct dynamical problem of seismics for horizontally stratified medium," Siberian Electronic Mathematical Reports, vol. 2, pp. 23-61, 2005, http://semr.math.nsc .ru/v2/p23-61.pdf. 


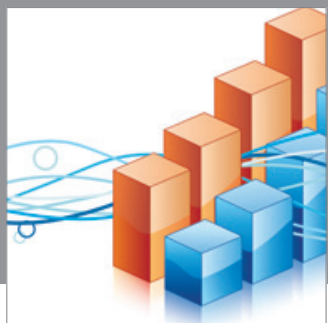

Advances in

Operations Research

mansans

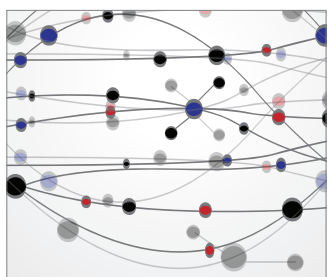

The Scientific World Journal
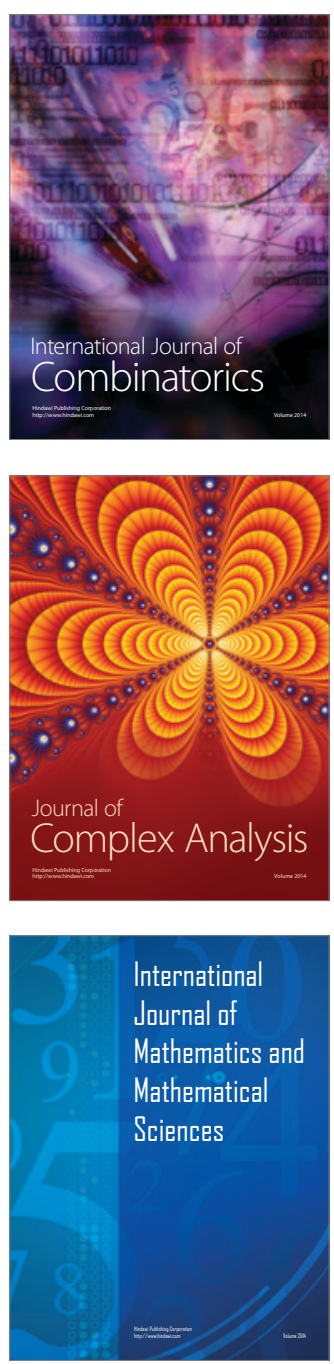
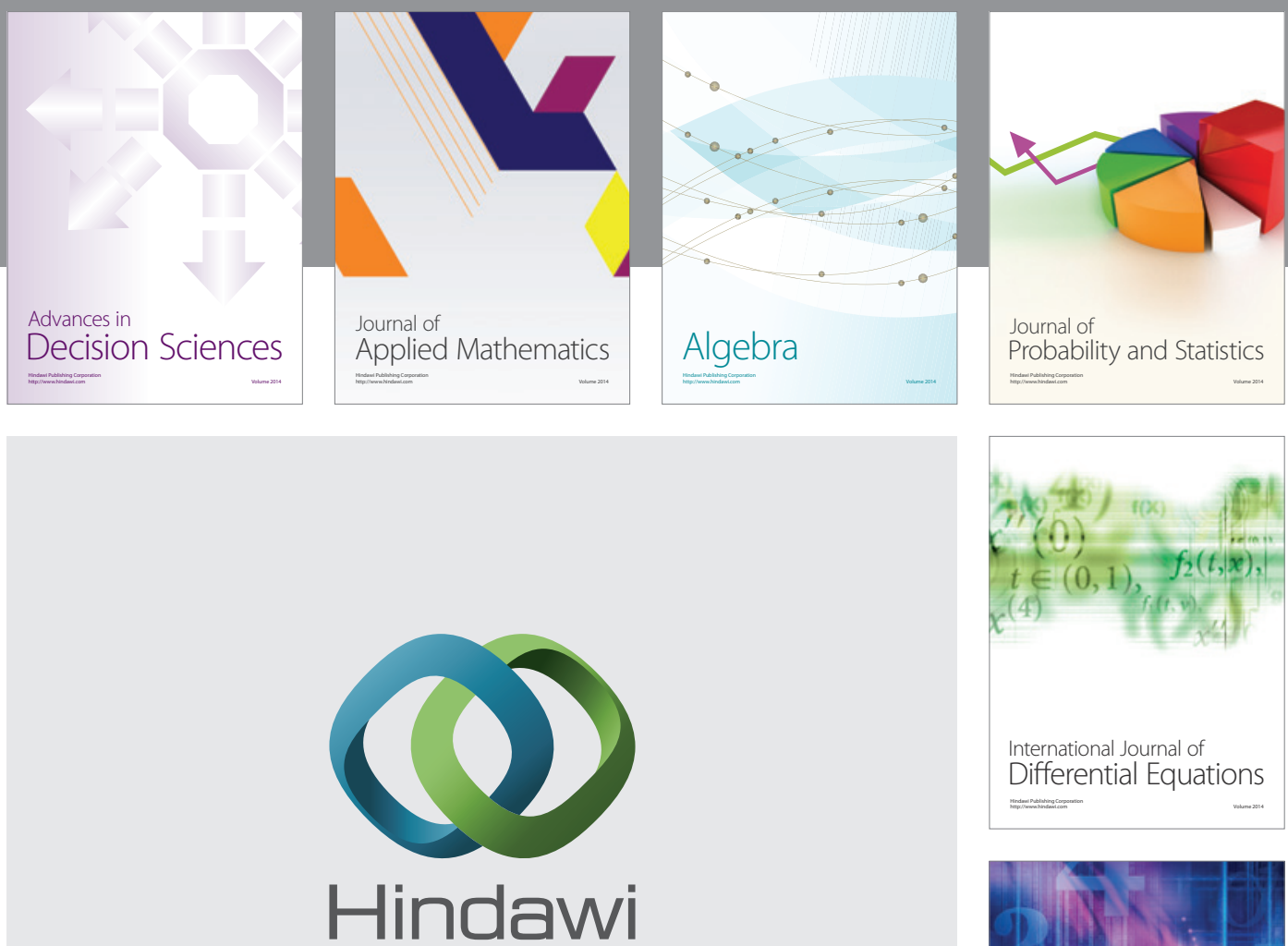

Submit your manuscripts at http://www.hindawi.com
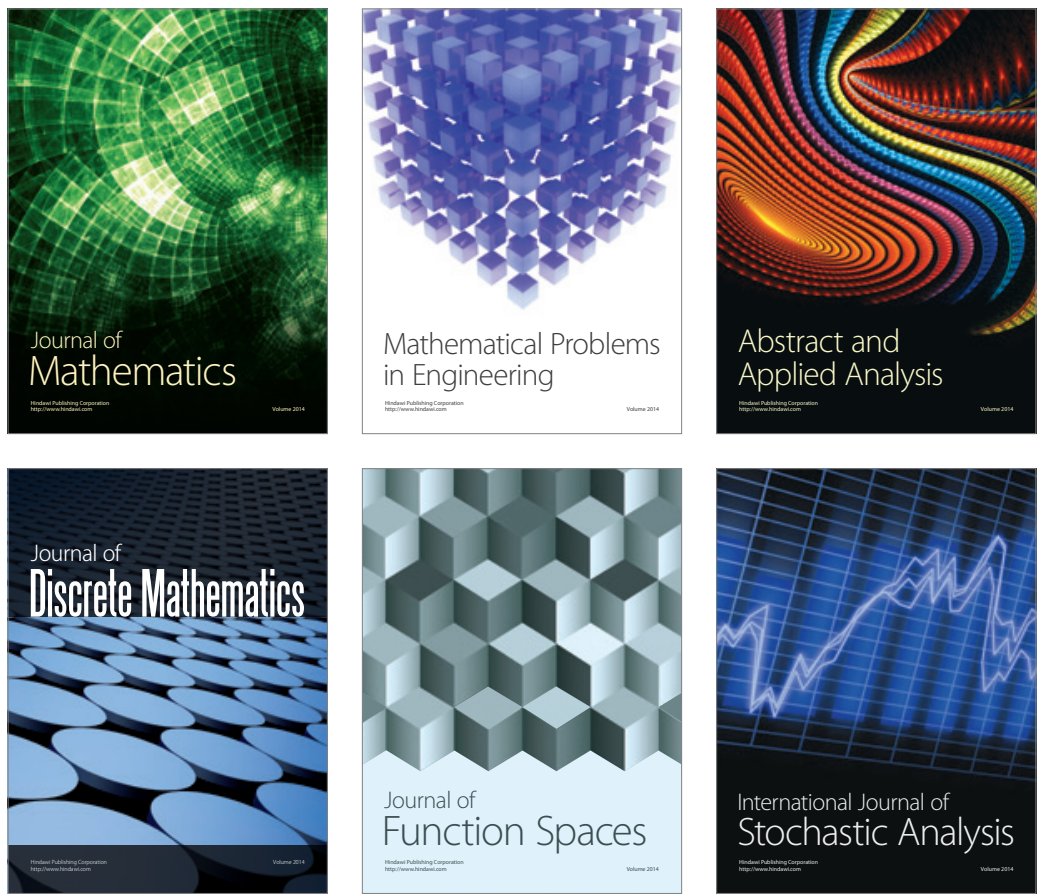

Journal of

Function Spaces

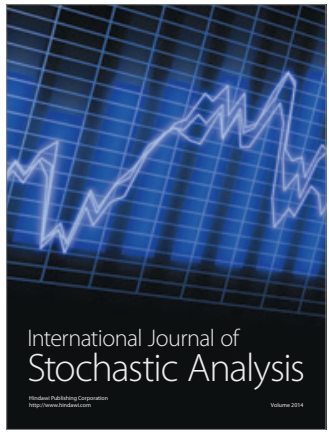

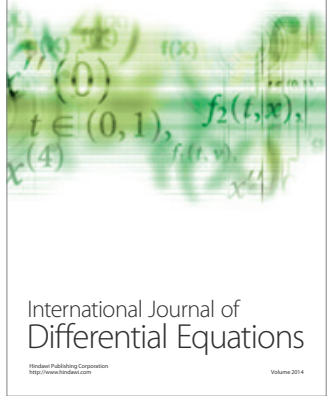
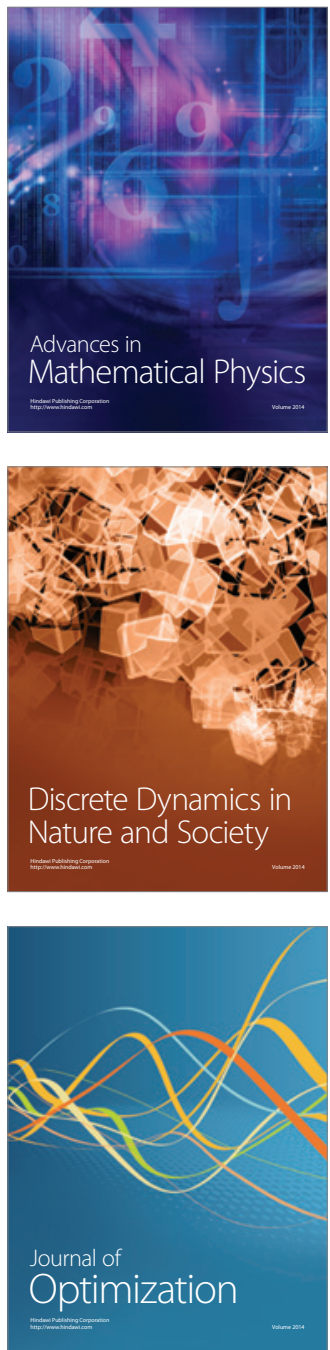